\title{
STRESS INOCULATION TRAINING TO CONTROL ANXIETY IN SPORT: TWO. CASE STUDIES IN SQUASH
}

\author{
R. MACE, DipPE, LCP, MA* and D. CARROLL, BSc, PhD** \\ *Newman College of Education, Birmingham B32 3NT \\ **Psychology Department, University of Birmingham
}

\begin{abstract}
This study arose as a result of two squash players, one male one female, seeking advice on how to improve their mental approach to playing. They both felt that their game suffered badly through too much anxiety. After preliminary interviews it was decided to use a programme of stress inoculation training to help them learn to control their anxiety. In order to obtain baseline measures of anxiety, both subjects completed a state anxiety questionnaire on five occasions, immediately prior to playing important league or team matches. They were then given eight training sessions. On completion of the training, subjects were asked to complete a further five state anxiety tests immediately prior to analogous matches. There was a considerable decrease in self-reported anxiety levels and both players reported that their performance had improved. It is argued that stress inoculation training is potentially very useful as a technique for controlling anxiety in certain competitive sports.
\end{abstract}

\section{INTRODUCTION}

There are a number of stress management techniques which can be used to help people relax. However, many of these are not appropriate for use in sport since there are many occasions when a high level of arousal will enhance performance. In the selection of a stress management programme therefore, the counsellor must have a clear understanding of the arousal/performance relationship (Martens, 1974; Landers, 1980; Landers, 1982). In the game of squash, for example, it is likely that a player will give his best performance when he/she is able to regulate arousal level to the optimum. Since optimum level varies according to the nature of the task and individual skill, it may be advantageous for players to learn to regulate their arousal levels for different shots and situations. The kill drive, for example, may be best played with a high level of arousal whereas for the high floating cross court lob the player should have a lower level of arousal. It seems appropriate, therefore, to train players not only to relax but also to learn to cope with high levels of arousal and, when appropriate, use the resultant higher drive to their advantage.

One stress management technique which allows for this is stress inoculation training (Meichenbaum, 1977). This has been used successfully both in basketball (Harrison and Feltz, 1981) and in abseiling (Mace and Carroll, 1985). Stress inoculation training is based on the principle of immunisation and allows subjects to learn to cope with increasing amounts of psychological stress. The procedure comprises three phases. The first phase is the education phase which gives subjects an understanding of how stress occurs. Recent research suggests that this phase is particularly important (Jaremko et al, 1980). Accordingly, in this study, subjects were also given an explanation of the arousal/performance relationship and its implications for sport. In the second phase, the rehearsal stage, the therapist trains subjects to use coping skills appropriate to their particular problem. Initially, this involves training subjects

\footnotetext{
Address for correspondence:

R. D. Mace

Newman College of Education

Genners Lane

Bartley Green

BIRMINGHAM B32 3NT
}

to use an appropriate relaxation technique. This is followed by a series of training sessions when subjects are taught to make coping self-statements in order to help them control their level of psychological stress. These sessions may also include training in visualisation and mental rehearsal. By the end of the treatment period subjects should be able to relax quickly, visualise specific situations and make positive self-statements e.g. "I am going to stay relaxed during the warm-up and study my opponent's technique". This part of the training is normally carried out in a quiet room with subjects either sitting or lying in their preferred position for relaxation and visualisation. Each session should last for approximately 45 mins with subjects having a number of attempts at visualising and making coping self-statements.

The third phase, the application phase, gives an opportunity for subjects to practise their coping skills in low/moderate stress situations. For example, in an experiment into the effect of stress inoculation training on anxiety levels prior to abseiling (Mace and Carroll, 1985), subjects were asked to practise using their coping selfstatements while abseiling from wall bars in a gymnasium. They were then asked to abseil from the roof of a 70' $(22 \mathrm{~m})$ building.

One of the major advantages of stress inoculation training is its flexibility. The procedure can be modified and applied to a wide variety of stress-related problems including anger control (Novaco, 1977) pain (Hackett and Horan, 1980) and fear (Mace and Carroll, 1985). It is this flexibility which makes stress inoculation training potentially very valuable for us in sport where competitors often suffer a loss of performance through stress arising from, for example, fear of injury or pain and fear of failure.

\section{CASE HISTORIES AND BEHAVIOURAL ASSESSMENT}

The descriptions below which shows how anxiety affected two squash players will be familiar to many who play competitive sport.

\section{The Case of Player $X$}

Player $X$ was a member of the third team of a Squash Club. During internal club league matches and friendly matches he consistently played to a higher standard than when he played for the team in representative matches. The 
increased importance of the match appeared to cause a considerable increase in anxiety associated with a below par performance. After a series of poor performances for the team he was dropped. He became disconsolate, played badly unimportant games and eventually decided to stop playing for a few months.

\section{The Case of Player $Y$}

Player $Y$ was a member of the women's team in the same squash club. She reported high levels of anxiety before important matches. This was becoming an increasing problem and she felt that her game suffered badly as a result. Although she tried very hard to relax she found that it was impossible for her to do so. Her form in internal league matches was usually much better than in representative matches.

During the preliminary interviews both subjects reported similar symptoms prior to important games, i.e. 'butterflies' in the stomach, general bodily tension and a feeling of nervousness. The effect of their performance was also similar, drives were over hit and shots were snatched. In addition, a poor shot elicited intense feelings of anger and frustration. In view of the similarity of the players' problems and the value of training subjects in pairs or small groups (Meichenbaum, 1972) it was decided to train both subjects together.

Prior to the stress inoculation training programme baseline measures of anxiety were obtained by administering a state anxiety questionnaire (Spielberger et al, 1970) on five occasions. Subjects completed the questionnaire immediately prior to playing in matches which they considered to be important and were likely to cause some anxiety beforehand e.g. ladder and league matches. Following intervention subjects were administered a further five state anxiety questionnaires which they completed immediately prior to similar matches.

\section{TREATMENT}

The first session was the education phase. Subjects were given a talk on how stress occurs and the relationship between arousal and performance in sport. They were encouraged to describe their own feelings and experiences when they were very anxious before and during matches. The remaining seven sessions comprised a series of progressive stages during which subjects were trained to relax, mentally rehearse specific shots, visualise specific situations and to make positive self-statements, e.g.

"See yourself warming up... tell yourself to play a few drives, feel yourself driving down the forehand wall... concentrate on the feel of the action ... now tell yourself to remember to study your opponent ... . tell yourself to play a variety of returns and watch his action.

Now see yourself getting ready to start the match. Go to your towel. Tell yourself you are going to enjoy the game ... you can control your emotions... tell yourself you feel good.

Now see yourself playing a match ... see yourself go in for a cross court kill drive ... feel yourself snatch at the ball and hear it crash into the tin ... feel yourself tense up ... feel yourself getting really annoyed with yourself ... now tell yourself to stay cool ... relax, tell yourself that shot was in the past...tell yourself not to worry about it...tell yourself to concentrate on the next point.

Now see yourself at the end of the first game ... go to your towel. Tell yourself to think about the good shots you played ... . tell yourself to keep concentrating ..."

In the latter training sessions, the application phase, subjects were given the opportunity to practise their coping skills in low/moderate stress situations. Each subject played squash against the counsellor who helped them to practise their self-instructions in the playing situations similar to those visualised. They then played a series of games during which an attempt was made to progressively increase the level of psychological stress experienced by the subjects. This was done by arranging for them to play matches against opponents who were not quite so good at squash and telling the subjects that if they lost they had to pay their opponent a small sum of money. By introducing a handicap system it became increasingly difficult for them to win. Throughout this series of games subjects practised applying their coping skills.

\section{RESULTS}

Both subjects responded well during the stress inoculation training programme. During the education phase they showed a lively interest in the arousal/performance relationship and were obviously convinced of the value of learning to control psychological stress in sport. Training sessions during the rehearsal phase went smoothly, both subjects worked enthusiastically and conscientiously at the relaxation technique and at making coping self-statements. On occasions, during the initial mental rehearsal training sessions, the subjects experienced difficulty in visualising a specific shot. This problem was resolved by asking them to visualise an expert playing the shot. The application phase appeared to be successful. Subjects were able to use their coping skills and they both reported that between games it was important to develop habits such as going to their towel. An act such as this became a stimulus for them to use their self-statements.

Following the training programme both subjects returned to active competition. They both reported that they experienced lower levels of anxiety and felt confident that they could cope if they became nervous. Player $X$ reported a considerable difference in his feelings after playing a poor shot. Prior to intervention he felt angry with himself and became tense. Following stress inoculation training, he used coping self-statements after playing badly and tried to concentrate on the next point. Player $Y$ reported that she gained most benefit from the training by being more relaxed prior to a match. She also thought that the use of self- statements between games was particularly valuable in controlling her anxiety. After a series of consistent performances Player $X$ regained his team place. Player $Y$ reported an increasing confidence in league and team matches. A few weeks after the stress inoculation training programme she entered the women's club championships and despite being ranked fifth, won the individual trophy. Further, for anxiety measures, both subjects were matched against two control subjects who also completed the state anxiety questionnaires on five occasions. These two did not display similar state anxiety reductions.

\section{DISCUSSION}

Both players were of the opinion that the training 
TABLEI

State anxiety mean scores (mean values) derived from the questionnaires administered immediately prior to playing in five different matches

\begin{tabular}{lcc}
\hline & Pre-intervention & Post intervention \\
\hline Player X & 58.0 & 44.6 \\
Player Y & 45.6 & 30.6 \\
Control subject A & 43.2 & 42.0 \\
Control subject B & 60.3 & 68.0 \\
\hline
\end{tabular}

programme was very valuable and had improved their performance. However, performance was only assessed subjectively and it cannot be concluded that the training procedure was responsible even if they were playing better after intervention. Self-reported anxiety levels indicated that the training was associated with a lower state anxiety immediately prior to playing. This was not the case for the two control subjects.

It can be argued, therefore, that while the present observations do not establish unequivocally stress inoculation as the instrument of change in this context, they are certaily consistent with the view that it is a useful technique both for reducing anxiety and augmenting performance in certain competitive sports.

\section{References}

Hackett, G. and Horan, J. J., 1980 "Stress inoculation for pain: what's really going on?" Journal of Counselling Psychology 27: 107-116.

Harrison, R. P. and Feltz, D., 1981 "Stress inoculation for athletes: Description and case example". Motor Skills: Theory into practice 5 (1): 53-61.

Jaremko, M. E., Hadfield, R. and Walker, W. E., 1980 "Contribution of an educational phase to stress inoculation of speech anxiety". Perceptual and Motor Skills 50: 495-501.

Landers, D., 1980 "The arousal performance relationship revisited". Research Quarterly for Exercise and Sport 51: 77-90.

Landers, D., 1982 "Arousal, attention and skilled performance; further considerations". Quest 33 (2): 271-283.

Mace, R. D. and Carroll, D., 1985 "The control of anxiety in sport: stress inoculation training prior to abseiling". International Journal of Sport Psychology 16: 165-175.

Martens, R., 1974 "Arousal and motor performance". In: J. H. Wilmore. Exercise and Sport Science Reviews (Vol. 2), New York, Academic Press.

Meichenbaum, D., 1972. Therapist Manual for Cognitive Behavior Modification. Unpublished manuscript. University of Waterloo, Ontario, Canada.

Meichenbaum, D., 1977. Cognitive Behavior Modification. New York: Plenum Press.

Novaco, R., 1977 "A stress inoculation approach to anger management in the training of law enforcement officers". American Journal of Community Psychology 5 (3): 327-346.

Spielberger, C. D., Gorsuch,R. L. and Lushene, R. E., 1970 "S.T.A.I. Manual for the State-Trait Anxiety Inventory. Palo Alto, California, Consulting Psychologists' Press.

\section{BOOK REVIEW}

Title:

Authors: James M. Booher and Gary A. Thibodeau

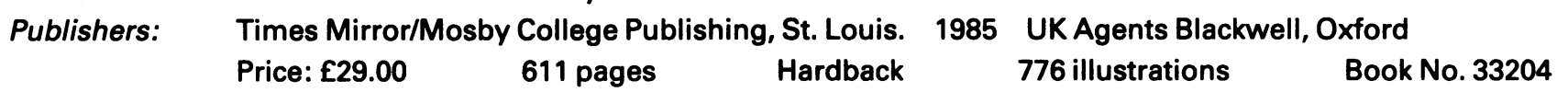

This book must be seen for what it is - a text specifically for trainee athletic trainers in the USA. British readers will be interested to read that "As a sports medicine subspeciality, athletic training provides a wide array of health care support services for athletes. Athletic training (is) 'the art and science of prevention and management of injuries at all levels of athletic activity.' The provider of athletic training services is an athletic trainer." In other words, we are reviewing a textbook for some sort of sport-based paramedic whose function falls somewhere between physiotherapists and doctors as understood in UK. Not one word concerns a function understood in Britain as training in the sense of coaching.

Successive chapters cover the functional anatomy and clinical examination (coyly rephrased throughout as 'evaluation' or 'injury assessment process', presumably to avoid offence to real doctors) of the locomotor system - bones, joints, muscles and the regions. There are chapters on injury mechanisms, and accounts of most injuries, usually with excellent diagrams and clinical photos. Clinical examination techniques are well described and illustrated.

There are a number of detailed criticisms by way of omission, e.g. stress fractures of the hip, or anywhere else in the detail expected in a book of such length. The accounts of Achilles injury and shin pain are totally inadequate; the deliberate evasion of discussion of footwear to this audience is bizarre, especially as there follows a good picture of worn shoes and an account of metatarsalgia without mention of footwear causes. Muscle tears are not differentiated well, sports causes of many conditions described, e.g. tennis elbow, are not discussed.

Perhaps the main criticism is that the book does not seem to define its level properly. On the one hand it has a good illustrated account of locomotor system examination; on the other hand it omits the sort of clinical detail which this paramedical audience craves. That the book invites such criticism is clear from, for example, the irrelevant (in this context) detailed descriptions of radiological techniques usurping 22 pages in the 29 page chapter on 'Factors relating to injury assessment'. Whether this represents some political confusion, I do not know; but it spoils a book with considerable merits.

As so often, the reader may ask why over 600 pages are needed for such a book. Gratuitous tautology must make life difficult for the American student! One copy for the college library should be worthwhile, but until drastic editing and revision make it the classic it could become, I would not burden students with its size. 